\title{
MEMORIAS
}

\section{Seminario de Actualización en Neurorehabilitación en Pediatría ${ }^{1}$}

Especialización en Fisioterapia en Neurorehabilitación 29 de Noviembre de 2018

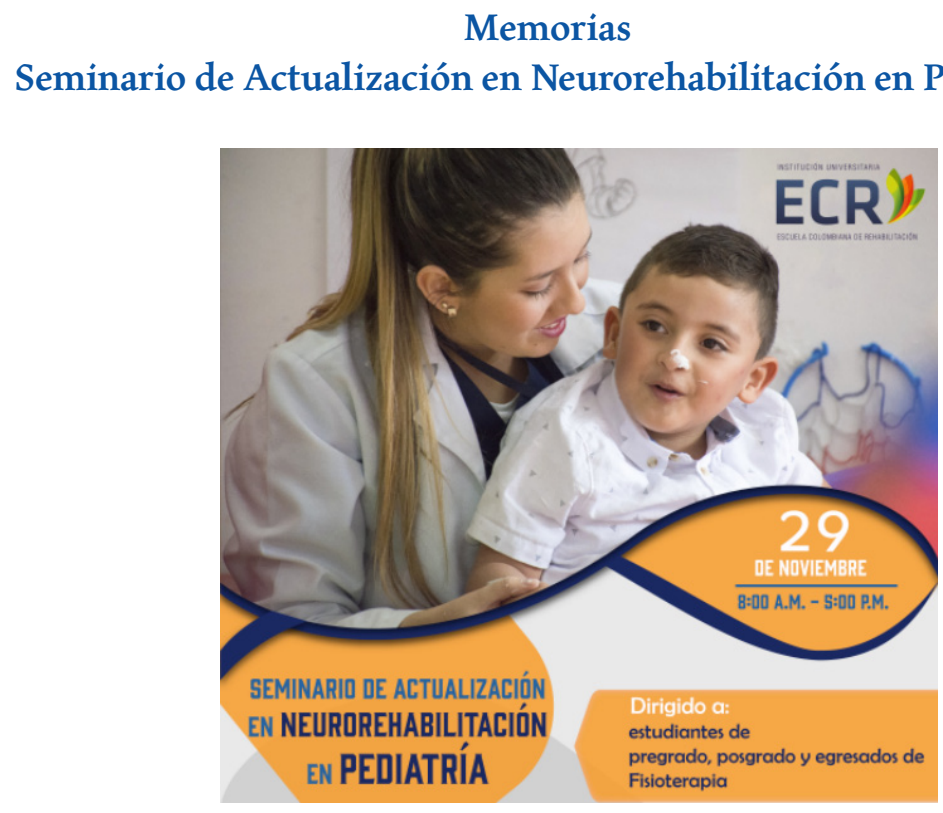

Especialización en Fisioterapia en Neurorehabilitación

Noviembre 29 de 2018

\section{Presentación}

Con el propósito de facilitar que los especialistas profundicen en la comprensión integral de las condiciones de salud, a partir del análisis del control y el aprendizaje motor para el manejo de individuos y colectivos, la Especialización en Fisioterapia en Neurorehabilitación de la facultad de Fisioterapia de la Escuela Colombiana de Rehabilitación, organizó el Seminario de Actualización en Neurorehabilitación en Pediatría que se llevó a cabo en la ciudad de Bogotá el día 29 de noviembre del 2018. Este documento contiene los resúmenes de las ponencias presentadas en dicho evento.

El objetivo principal del Seminario fue proporcionar actualización en las nuevas tendencias en neuropediatría. Bajo esta sombrilla se propuso integrar los conceptos teóricos con las experiencias investigativas desarrolladas en el transcurso del posgrado, presentar los últimos avances en la neurorehabilitación para población pediátrica y exponer algunos productos investigativos derivados del posgrado de Fisioterapia en Neurorehabilitación. En total se presentan diez resú-

\footnotetext{
Evento organizado por la Especialización en Fisioterapia en Neurorehabilitación bajo la coordinación de la Ft. Esp. y MSc. en Neurorehabilitación Cyndi Yacira Meneses Castaño.
} 
menes de ponencias (nueve nacionales y una internacional), dos de ellas corresponden a productos generados desde la especialización.

\section{PONENCIAS CENTRALES}

\section{Neurocriminología - jóvenes infractores y salud mental José Julián Javela}

El ponente inicia su presentación mostrando el papel de las neurociencias en la explicación de la conducta criminal y una reseña histórica donde se identifica neuroanatómicamente la criminología, trayendo a autores como Carl Sagan, Suzana Herculano-Houzel y llegando a Cesar Lombroso que habla sobre cómo desarrollos embrionarios y procesos evolutivos incompletos y erróneos podían derivar en criminales natos.

Se presentó el concepto de criminología que es la ciencia empírica e interdisciplinaria que surge a principios del siglo XIX y que tiene como objeto el estudio del delincuente, el lugar de los hechos, el delito, las conductas desviadas, y el control social con relación al delito mismo siguiendo las bases del método científico. Por su parte, la neurociencia es una disciplina que incluye muchas ciencias que se ocupan de estudiar, desde un punto de vista inter, multi y transdisciplinario la estructura y la organización funcional del Sistema Nervioso.

De acuerdo con lo anterior, se definió la neurocriminología como el campo de estudio que busca aplicar las técnicas y principios de la neurociencia para mejorar el entendimiento del crimen o el delito (Glenn \& Raine, 2014). La neurocriminología es una subdisciplina de la criminología que aplica técnicas de Neurociencia para determinar si hay un problema neurobiológico en un crimen o determinantes mentales de la criminalidad, para intentar evitar el delito o su reincidencia, y para aplicar las penas más justas y eficaces para castigar al delincuente y propiciar su reinserción.

En su desarrollo, la ponencia abarcó temáticas relacionadas con agresión, conducta delictiva y, violencia y los métodos diagnósticos para detectar anomalias relacionadas. Adicionalmente, se presentó el análisis de la genética, hormonas y neurotransmisores, psicobiología, imágenes cerebrales - neurología y neurología e interacción química y su relación con las conductas criminales.

A manera de conclusión se presentó que "Los psicópatas saben lo que está bien y lo que está mal, pero no tienen un sentimiento de qué está bien y qué está mal”. Es decir, saben la regla, pero no la interiorizan, no presentan un sentimiento de angustia cuando saben que van a romperla, tienen menor miedo al castigo y menos emoción positiva o negativa a la hora de hacer el bien o hacer el mal.

Por último se presentaron algunos retos de la neurocriminología:

- Analizar las influencias específicas de la genética y el contexto que inducen cambios neurobiológicos que resultan en un riesgo cognitivo, afectivo y conductual para la violencia.

- Desarrollar programas de intervención innovadores y adecuados para la prevención de la criminalidad en estas personas.

- Potenciar y mejorar los métodos predictivos de la criminalidad para así dar resultados más precisos y válidos con rigor científico desde las Neurociencias.

- Evidenciar el papel crucial que juega la neurofisiología de un individuo a la hora de cometer un delito.

- Explicar desde el campo de acción de las neurociencias cognitivas y básicas el comportamiento criminal e ilegal.

\footnotetext{
2 Psicólogo, PhD en Neurociencias. Actualmente se desempeña como docente en la Universidad Católica de Pereira y es investigador colaborador en la Universidad Complutense de Madrid.
} 
- Desarrollar un acercamiento a los principales factores psicobiológicos relacionados con la agresión y la violencia.

- Brindar conocimientos actuales sobre la contribución de las alteraciones anatómicas en el funcionamiento del cerebro a la conducta violenta y la criminalidad.

- Aplicar técnicas de neurociencia para determinar si hay un problema neurobiológico en un crimen o determinantes mentales de la criminalidad, para intentar evitar el delito o su reincidencia y para aplicar las penas más justas y eficaces para castigar al delincuente y propiciar su reinserción

- Evaluación de los respectivos dominios cognitivos mediante la utilización de pruebas neuropsicológicas.

Neuropsicología en Pediatría

Ana María Vélez ${ }^{3}$

El objetivo de la neuropsicología es el estudio de la organización cerebral, de la actividad cognitiva-conductual, así como el análisis de sus alteraciones en caso de presentarse patología cerebral (Ardila \& Roselli, 2007). La neuropsicología del desarrollo, también llamada neuropsicología infantil o pediátrica, es una neurociencia que estudia las relaciones entre conducta y cognición de un cerebro en desarrollo. La neuropsicología del desarrollo pretende aplicar los conocimientos científicos de dichas relaciones, para evaluar y compensar las consecuencias derivadas de lesiones cerebrales producidas durante el embarazo, el parto y/o el transcurso de la infancia y adolescencia, dentro de un contexto dinámico que estudia las relaciones entre el cerebro y la conducta/cognición para un cerebro en desarrollo (Maduración).

La función cerebral superior se define como un sistema funcional complejo, es decir, las funciones no pueden localizarse en zonas restringidas de la corteza cerebral o en grupos de células aislados, "sino que deben estar organizados en sistemas de zonas que trabajan armónicamente, cada una de las cuales ejerce su papel dentro del sistema funcional complejo, y que pueden estar situadas en áreas completamente diferentes, y a menudo, muy distantes del cerebro".

A continuación la ponente introduce el concepto de rehabilitación neuropsicológica que se encarga del tratamiento o manejo de los problemas cognitivos, de conducta, emocionales o las dificultades adaptativas del niño o adolescente, que previamente haya sido valorado por un neuropsicólogo clínico infantil. Este abordaje se hace de forma global, abarcando tanto las dificultades cognitivas, conductuales y emocionales, como la situación familiar, escolar y social del niño o adolescente. También presenta las características para determinar si un niño presenta problemas en su neurodesarrollo. Curiosamente en Colombia, siendo la población infantil la más vulnerable a situaciones traumáticas (maltrato, abuso, explotación, negligencia), no es fácil encontrar estudios relacionados con los procesos madurativos del desarrollo neuropsicológico en la infancia.

Adicionalmente, se presentan los conceptos de la evaluación del neurodesarrollo infantil y la definición del concepto de "trastornos del neurodesarrollo", se hace un análisis de los procesos de maduración cerebral y el desarrollo cognitivo en donde se muestra que el desarrollo del Sistema Nervioso es secuencial, extiende su desarrollo en la interacción con el medio ambiente y con eventos genéticamente programados.

Por último, se presenta el concepto de "apoptosis" como mecanismo de poda neuronal y se presenta la razón del por qué la muerte celular en una fase crucial para el desarrollo del encéfalo.

\footnotetext{
3 Especialista y magister en Neuropsicología clínica. Actualmente trabaja en Uniepilepsias, es docente de la Universidad iberoamericana y cooordina los laboratorios de neuropsicología de la universidad Santo Tomas.
} 
Deporte como estrategia de inclusión en pacientes pediátricos Felipe Herrera ${ }^{4}$ (Video conferencia Chile)

El ponente incia hablando sobre la baja actividad física como problemática mundial, y especialmente regional pues América Latina y el Caribe es una de las más afectadas. El problema es particularmente agudo en los países pobres y con mayor desigualdad, y entre las poblaciones pobres y menos escolarizadas de cada país. La actividad física también se ve aquejada por una marcada diferencia entre los géneros: las mujeres hacen menos ejercicio que los hombres. La inactividad física entre los jóvenes y niños en edad escolar resulta particularmente preocupante. No obstante, este panorama de una región con una población cada vez más obesa y sedentaria es incompleto e impreciso debido a la falta de datos fiables y comparables. Parte de la asignatura pendiente es recoger más y mejores datos sobre sedentarismo y actividad física en la región.

La mayoría de las personas sabe que le conviene hacer ejercicio, tanto individualmente como en grupo. La actividad física no solo previene enfermedades y promueve una vida sana, sino que también mejora el bienestar mental y la interacción social, y contribuye al desarrollo económico en diferentes contextos geográficos, culturales y políticos. Lamentablemente, las personas ya no son tan activas como antes. De hecho, la inactividad física ha sido reconocida como una pandemia que exige la adopción de medidas a nivel mundial (Das \& Horton, 2016). A partir de datos auto-reportados, se calcula que en 2016 la prevalencia mundial de inactividad física en adultos, definida como la falta de 150 minutos de actividad de intensidad moderada o 75 minutos de actividad vigorosa por semana (o una combinación equivalente), ascendía a cerca del 24\% (Das \& Horton, 2016). Según datos de la Organización Mundial de la Salud (OMS), la región de América Latina y el Caribe parece seguir esta tendencia global, con una prevalencia de inactividad física en adultos estimada en el $32 \%$. América Latina y el Caribe es la segunda región con mayor inactividad física del mundo, tan solo detrás de la región de las Américas en su conjunto, de la cual también forma parte.

Debido a la falta de evidencia y de consenso con respecto a lo que funciona y lo que no, así como a la importancia de diseñar correctamente los programas, lo primero que se recomienda es incrementar la recolección de datos, empezar con proyectos pequeños que puedan evaluarse, y ampliarlos una vez que se tenga la certeza de que por lo menos no producen ningún daño. Habida cuenta de que es imprescindible mejorar las habilidades motoras de los niños y sus conocimientos sobre la actividad física, así como también aumentar los niveles de ejercicio en niños y adultos, las intervenciones de economía del comportamiento de eficacia comprobada ofrecen una herramienta valiosa.

El nudging puede aumentar la actividad física con pocas repercusiones negativas, a la vez que proporciona tiempo para aprender en qué se puede usar mejor el dinero. Por último, debido a que las inversiones en otras áreas podrían repercutir en la actividad física, es importante interiorizar esto de la misma manera en que se ha tomado conciencia acerca del papel de la política pública en el medio ambiente y otros ámbitos. Por ejemplo, el diseño urbano y la infraestructura urbana afectan la actividad física. De por sí, tanto las inversiones en vías y transporte como el código de construcción, deberían considerar el efecto que ejercen sobre la actividad física, al igual que tratan de hacerlo con respecto a las preocupaciones ambientales. Por suerte, ambos van de la mano y se refuerzan mutuamente.

Por último, el ponente destaca frases alucivas a la importancia del deporte:

"El deporte tiene el poder de cambiar el mundo. Tiene el poder de inspirar, tiene el poder de unir a las personas como muy pocas otras cosas". Nelson R. Mandela.

"El deporte es un lenguaje universal que puede unir a las personas, sin importar su origen, sus creencias religiosas o su situación económica”. Kofi Annan, Secretario General de la ONU.

${ }^{4}$ Kinesiólogo docente de la Universidad Viña del Mar (Chile) 
"El deporte ha cambiado las actitudes y la cultura en formas que parecen muy sutiles pero que, en última instancia, nos han hecho modificar nuestra concepción sobre nosotros mismos y nuestro pasado". Barack Obama, expresidente de Estados Unidos.

"Mucho más que simple rendimiento, el deporte es sinónimo de educación y responsabilidad. Inculca valores firmes en los jóvenes, que los ayudan a crecer”. Pernilla Wiberg, una de las más exitosas esquiadoras de la década de 1990

\section{Análisis de Marcha en Rehabilitación Pediátrica}

\section{Andrea Garzón ${ }^{5}$}

La ponente inicia haciendo un recorrido por el análisis de movimiento corporal humano, marcando como punto de referencia a Edward James Muggeridge y sus comparaciones con la ejecución de los equinos a la marcha humana, seguido de ello, hace énfasis en el análisis computarizado de la marcha y presenta conceptos de macha como el de Perry (2010) quien indica que es una secuencia repetitiva de movimientos de las extremidades inferiores para mover simultáneamente el cuerpo hacia adelante, mientras también se mantiene la estabilidad y la postura.

Posteriormente, hace un recorrido por cada uno de los principales elementos de la marcha como son: el sistema de control, la fuente de energía, palancas y fuerzas; de esta forma, presenta un análisis del ciclo de la marcha.

A continuación, desarrolla la temática de la valoración de la marcha en niños donde se dan algunos elementos claves para tener en cuenta y se presentan las limitaciones de este proceso. Adicionalmente se presentan las alteraciones y causas de las mismas.

Por último, se hace referencia al análisis computarizado de la marcha el cual permite, de manera detallada, entender las alteraciones de la marcha (cinética y cinemática) con importantes referentes como la electromiografía y la podobarografía, el cual es el análisis de la distribución de cargas de manera estática y dinámica con el fin de:

- Entender las alteraciones dinámicas de la marcha y diferenciar las causas primarias, las secundarias y los mecanismos compensatorios.

- Definir el comportamiento de la marcha en diferentes patologías.

- Ayudar a planificar tratamientos e intervenciones optimizando los recursos (Quirúrgicos-No quirúrgicos).

- Aporta información para la toma de decisiones con objetivos cuantificados.

- Medir resultados de tratamientos.

- Hablar un lenguaje común y permite intercambiar experiencias.

- Facilita la investigación.

Importancia de la Actividad física, ejercicio físico y deporte en las habilidades cognitivas en la Infancia Fabiola Gonzales ${ }^{6}$

La ponente inicia hablando sobre la importancia de las funciones ejecutivas en la actividad física. Las funciones ejecutivas son el conjunto de procesos mentales para controlar, regular y planear. El modelo neuropsicológico de funciones frontales y ejecutivas tiene diferentes niveles jerárquicos; dentro del nivel básico encontramos las funciones frontales básicas (COF corteza orbito frontal y CFM corteza frontomedial), el segundo nivel se encarga de la memoria de trabajo (CPFDL corteza prefrontal dorso lateral), el tercer nivel referencia las funciones ejecutivas de la (CPFDL corteza prefrontal dorso- lateral) y el nivel cuatro que se relaciona con las metafunciones (CPFA corteza prefrontal anterior) encargada

\footnotetext{
5 Candidata a Magister en bioingeniería en la Universidad del Rosario. Actualmente se desempeña como fisioterapeuta en Mobility Group.

${ }^{6}$ Especialista en ejercicio físico y Magister en Actividad Física y Deporte de la Universidad Nacional de Colombia. Actualmente es docente del programa de fisioterapia de la ECR.
} 
de la metamemoria, comprensión de sentido figurado y la abstracción.

A continuación presenta conceptos clave como control inhibitorio, memoria de trabajo, flexibilidad mental y planeación. A partir de esto, muestra la relación de las funciones ejecutivas con la aptitud física, que se conforma en primaria y secundaria y parte de regular o inhibir un comportamiento, actividades multitareas y evitar la distracción así como las capacidades cognitivas del nivel superior.

La ponente presenta una serie de análisis como:

- La cognición versus la actividad física que se relaciona con el aprendizaje para el desempeño académico en asignaturas (matemáticas, inglés, lectura crítica y ciencia) y funciones ejecutivas para el control inhibitorio, memoria de trabajo, flexibilidad mental y planeación, utilizando herramientas como la acelerometría y el fitness gram.

- Los niveles de aptitud física y el rendimiento académico, se registra y compara combinado con pruebas neurocognitivas desde donde se encamina la importancia del aprendizaje inhibición (comprensión de contextos inglés ciencias, alfabetización y habilidades matemáticas) y memoria de trabajo (comprensión lectora, proceso razonamiento y habilidades lingüísticas).

- La planeación para logro de (meta en un menor tiempo, esfuerzo y dispersión cognitiva) y tareas cognitivas de cualquier índole.

- La flexibilidad mental con el fin de identificar y generar patrones, flexibilidad para usar conocimientos y adaptarlos, y uso de matemáticas, características determinadas o que se evidencian con pruebas como PISA, pruebas saber o rendimiento escolar.

La ponente recalca la importancia de la relación deportiva con las pruebas en áreas como la matemática y sus resultados para mejorar la atención y memoria de trabajo espacial. También se plasma diferentes razones de tipo neurofisiológicas de las funciones ejecutivas y el ejercicio físico, el cual favorece la reparación neuronal y la plasticidad de la arquitectura cerebral, promoviendo factor de crecimiento neural, factor neurotrófico derivado del cerebro y las neurotrofinas.

Los resultados apoyan los hallazgos documentales en relación con la importancia asociativa que tiene los niveles aptitud física con el desempeño en las tareas ejecutivas como lo son la inhibición, la memoria de trabajo y la planeación secuencial. Por otro lado, las asociaciones positivas en variables propias de la actividad física y el rendimiento cognitivo, ofrece información a considerar desde las instituciones educativas y entes gubernamentales para favorecer la promoción del ejercicio físico en todo el ciclo escolar (Muralidharan \& Prakash, 2017). Y por último, el ejercicio físico es un método sencillo y de bajo costo para tenerse efectivamente en cuenta como una estrategia precursora, no solo en la prevención de ECNT sino en el desarrollo cognitivo, interpretado en mejor rendimiento de los Adolescentes a nivel escolar y social.

\section{Importancia del proceso de evaluación fisioterapéutica en neurorehabilitación}

Liliana Maya ${ }^{7}$

La ponente inicia su intervención hablando de los elementos de la guía de atención fisioterapéutica: examen, evaluación, diagnóstico, pronóstico, intervención y logros, y se refiere a la importancia del proceso evaluativo en el diagnóstico en neurorehabilitación.

Posteriormente muestra la relación con los periodos históricos mundiales de la Fisioterapia a lo largo de cuatro etapas: Primera etapa, centrada en la disfunción de los sistemas musculo-esqueléticos y neuromuscular periférico; segunda etapa, centrada en la disfunción del sistema nervioso central; tercera etapa ,centrada en la disfunción articular y el desarrollo de la terapia manual y cuarta etapa (actual), centrada en el sistema del movimiento enfocada en la examinación, evaluación, diagnóstico, pronóstico, intervención y resultados. 
Hace un desarrollo de la fase de diagnóstico, en el cual se presenta tanto un proceso como una descripción. El proceso diagnóstico incluye la evaluación e integración de los datos que se obtienen del examen con el propósito de guiar el pronóstico, el plan de cuidado y las estrategias de intervención. El fisioterapeuta asigna descriptores diagnósticos que identifican una condición o síndrome al nivel de sistema, especialmente el sistema humano de movimiento y al nivel de toda la persona.

Por último, manifiesta que existen unos retos importantes como realizar investigación sobre la relación de la fisioterapia y la ciencia cognitiva, para conocer las implicaciones del enactivismo y las teoría sensorio-motoras en el movimiento corporal humano, que nos permita comprender diferentes tipos de fenómenos, esto es, cuando un paciente tiene una falta de conocimiento sobre cómo moverse (para actuar con el propio cuerpo), cómo se da la alteración para percibir el lazo de percepción-acción y la capacidad para predecir; los cuales son procesos importantes de aprendizaje motor.

Otro reto es encontrar maneras de ver nuestro conocimiento y construcción del mismo, en un sentido más amplio con evidencia de múltiples fuentes. Según Shaw y DeForge (2012) el fisioterapeuta es un "bricoleur", el término "bricoleur" se refiere a un hombre o mujer de "mantenimiento" que utiliza todas las herramientas y tipos de conocimiento disponibles. La noción de fisioterapeutas como "bricoleurs" reconoce que todo el conocimiento práctico se sitúa dentro de contextos sociales, culturales e históricos que dan forma a nuestras creencias sobre lo que cuenta como conocimiento de fisioterapia. Este reconocimiento lleva a los fisioterapeutas a que actúen como "bricoleurs" al abrazar múltiples epistemologías, descubriendo nuevas formas de conocimiento y estrategias de razonamiento clínico para proporcionar un enfoque más holístico de la práctica fisioterapéutica.

Modelos animales y epilepsia, control de crisis epilépticas

José Julián Javela ${ }^{8}$

El ponente inicia hablando sobre la epilepsia y su definición como una enfermedad crónica con predisposición a generar crisis epilépticas, el mecanismo básico de la epileptogénesis radica en la pérdida del balance entre la excitación/ inhibición. Su origen radica en estructuras mesio-basales (hipocampo, amígdala y corteza entorrinal).

De acuerdo a los estudios epidemiológicos, 0.5-2 \% de la población padece de epilepsia, y la Epilepsia del Lóbulo Temporal es la más frecuente con una resistencia al tratamiento es del 30\%

De los modelos animales de tratamiento para la epilepsia se encontró:

- Kindling eléctrico: Estimulación en amígdala/hipocampo.

- Kindling químico: Picrotoxina, bicuculina, pentilentetrazol (PTZ) (Javela, 2012).

- Status epilepticus: Acido kaínico, pilocarpina, 4-Aminopiridina y PTZ

El ponente habla sobre los recientes estudios que está ejecutando sobre el tema con el objetivo de caracterizar mediante la Tomografía por Emisión de Positrones (PET) el modelo de epilepsia in vivo por bloqueo de las interneuronas GABAérgicas mediante la administración de PTZ. Mediante el uso de un Equipo de micro PET.

Como resultados se obtuvo que la fase crítica de las crisis convulsivas inducida por el PTZ provoca un aumento del metabolismo cerebral, principalmente en tálamo, hipocampo y estriado. El descenso de la actividad neuronal durante la depresión post-ictal se correlaciona con un hipometabolismo generalizado en todas las estructuras cerebrales estudia-

\footnotetext{
8 Psicólogo, PhD en Neurociencias. Actualmente se desempeña como docente en la Universidad Católica de Pereira y es investigador colaborador en la Universidad Complutense de Madrid.
} 
das. Por último el PET es una herramienta válida que permite hacer observación en vivo de la actividad convulsiva. Así mismo, es una alternativa a los métodos tradicionales para valoración de la epilepsia.

\section{Abordaje desde la Hidroterapia en Neuropediatría}

Diana Tafur

La ponente inicia su diálogo exponiendo conceptos básicos como el proceso de diagnóstico y la importancia de crear objetivos claros para avanzar con buen rumbo a una meta, e introdue el concepto de Objetivos SMART: S specific (Específico), M measurable (Medible), A Achievable (Alcanzable), R Relevant (Relevante) T time - Bound (en tiempo). Dentro del concepto de intervención se plantean las diferentes estrategias dentro de las que están: cinéticas, cinemáticas, terapéuticas, multimodales, especializadas, artísticas, lúdicas y tecnología en rehabilitación. Adicionalmente se presenta el concepto de Re-aprendizaje a la Funcionalidad.

Se define también la hidroterapia como la utilización terapéutica del agua por sus propiedades físicas, que estudia la aplicación externa del agua sobre el cuerpo humano, siempre que sea con fines terapéuticos y principalmente como vector mecánico y térmico. Las bases físicas de la hidroterapia se sustentan en que el agua logra efectos terapéuticos al proporcionar una energía mecánica y/o térmica; con principios mecánicos como factores hidrostáticos, hidrodinámicos e hidrocinéticos, y principios térmicos como las aplicaciones termoterapeuticas (calor - frío).

Posteriormente presenta las aplicaciones terapéuticas de la hidroterapia haciendo énfasis en el concepto Halliwick (Martin, 1981), que fue desarrollado a principios de 1950 en una escuela llamada The Halliwick School for Crippled Girls para enseñar a los pacientes con discapacidad física a nadar y desarrollar independencia en el agua. Este cuenta con 10 pasos como base para establecer el programa de tratamiento, que son:

1. Ajuste mental y desapego

2. Control de rotación sagital/desapego

3. Control de rotación transversal/desapego

4. Control de rotación longitudinal/desapego

5. Control de rotación combinada/desapego

6. Empuje/inversión mental/desapego

7. Equilibrio en calma/desapego

8. Deslizamiento con turbulencia/desapego

9. Progresión simple/desapego

10. Movimiento básico de Halliwick/desapego

Por último presenta la aplicación de la CIF frente al concepto Halliwick encontrando que los beneficios en los dominios funcionales conlleva a:

1. Función respiratoria (ritmo y sincronía entre inspiraciones y espiraciones para facilitar el movimiento).

2. Movilidad tisular y articular (Disminución de la propiedad tixotrópica y cambios en la extensibilidad muscular).

3. Estabilidad articular (progresión en la posición articular, propiocepción y conciencia corporal).

4. Fuerza muscular (Core Stability con resultados en la musculatura dinámica de tronco).

5. Tono muscular (Movimientos rotacionales extensibilidad del tejido, movilidad articular y funcionalidad).

6. Resistencia muscular (Entrenamiento de los 10 puntos, aumento de la tolerancia al ejercicio).

7. Control del movimiento voluntario (Habilidades perceptivas y visomotoras que ofrecen el tiempo para la programación y ejecución)

8. Patrones de marcha (transferencias de las habilidades al medio, incrementos en fuerza y velocidad de los pasos).

\footnotetext{
9 Especialista en Fisioterapia en Neurorehabilitación de la ECR, actualmente se desempeña como neurorehabilitadora en mobility group.
} 
Alternativas de tratamiento fisioterapéutico desde el enfoque neurorehabilitador en parálisis cerebral infantil espástica

Natalia Andrea Rubio Ruda ${ }^{10}$

La ponente inicia hablando sobre los pasos preliminares para la sistematización de las experiencias que de acuerdo con Kitchenham (2004) consiste en: Selección de fuentes de información, ejecución, análisis, abstracción, síntesis, reporte y plasmar conocimiento.

A continuación presenta una descripción de morfología y fisiología cerebral de la recepción de estímulos sensitivos, la integración de la información nueva con la almacenada, toma de decisiones y ejecución de respuestas a través de la generación de actividades motoras.

Se presenta la caracterización y clasificación de la parálisis cerebral infantil (PCI) según la localización del evento (Hipóxico - isquémico) y otra clasificación según el grado de afectación en diplejía espástica, hemiplejía espástica y tetraplejía espástica.

La ponente presente las formas de intervención fisioterapéutica de Parálisis Cerebral Infantil Espástica (PCIE) que consisten en: prevención (Detección temprana y observación de fallas contextuales) y tratamiento integral (planificado y coordinado). Dentro de los métodos de tratamiento fisioterapéutico de PCIE desde la Neurorehabilitación se tienen: concepto de Bobath, método de integración sensorial y neuroplasticidad.

Se resaltan en la presentación los siguientes elementos:

- La complejidad de lesiones neurológicas ocasionada por PCI y las necesidades individuales de cada paciente, dificultan la generalización de recursos terapéuticos de eficacia comprobada.

- La eficacia del tratamiento está en comprender la organización funcional del sistema nervioso.

- En Colombia el nivel de publicaciones sobre las estrategias de intervención utilizadas por el fisioterapeuta en Neurorehabilitación es bajo.

- Por estar ampliamente documentados en la revisión realizada los métodos más investigados para la intervención en PCIE son el método Bobath, integración sensorial y concepto de neuroplasticidad.

Integración sensorial en neurorehabilitación con pacientes de trauma craneoencefálico (TCE) en UCI pediátrica Dayhan Andrea Lozano González ${ }^{11}$

La ponente inicia contextualizando a los asistentes sobre qué es el trauma craneoencefálico (TCE) el cual se estima que en el 2020 se convierta en una de las primeras causas de muerte y discapacidad en el mundo (Soto, Salinas \& Hidalgo, 2013). El TCE (trauma cráneo encefálico) genera deficiencia en la habilidad para desarrollar e integrar diferentes movimientos, afecta la etapa aguda a nivel vascular, neuronal, glial y sistema cardiorrespiratorio, funciones motoras, sensoriales y cognitivas y los componentes mecánicos de diferentes estructuras (Pozaa et al., 2012).

El concepto de integración sensorial es presentado como fundamental en la atención del TCE y las razones sobre el vacío en conocimiento sobre este concepto (Yague Sebastian, 2005). La ponente realiza un análisis de la literatura sobre este tema e introduce la relación entre la integración sensorial y la neurorehabilitación. Resulta de gran importancia el rol del fisioterapeuta como neurorehabilitador en un equipo integral en el manejo de pacientes en la UCI pediátrico, ya

\footnotetext{
10 Especialista en Fisioterapia en Neurorehabilitación de la ECR, se desempeña como fisioterapeuta en la clínica Santa Teresa y como docente en la Universidad Pedagógica y Tecnológica de Colombia.

${ }^{11}$ Especialista en Fisioterapia en Neurorehabilitación de la ECR. Actualmente se desempeña como neurorehabilitadora en el Hospital Universitario de la Samaritana
} 
que ayuda a que las acciones que se realicen vayan enfocadas a mejorar no solo los procesos físicos del niño, sino que, a través de su conocimiento del movimiento corporal humano y del funcionamiento del sistema nervioso, puede unificar ambos conocimientos y generar un plan de acción en el cual se logre la activación de procesos cognoscitivos, sensoriales y perceptivos; a través de lo cual, se logre una intervención temprana que active los procesos de neuromodulación del SNC para así mejorar la funcionalidad del paciente.

Sus principales conclusiones son:

- La neuroplasticidad permite un proceso de organización cerebral que ayuda a la adquisición de habilidades y destrezas especialmente en el paciente pediátrico, esto nos indica que el cerebro se adapta, y se reorganiza en condiciones patológicas, por ello la integración sensorial es de suma importancia, ya que potencializa la recuperación del paciente con trauma craneoencefálico

- Desde la UCI se comienza un trabajo integral para la recuperación y rehabilitación del paciente en el menor tiempo posible y así evitar secuelas que puedan alterar su calidad de vida, en muchos casos lo que se desea procurar, tanto si se trata de un TCE moderado o severo, que las secuelas sean menores a través de un proceso de rehabilitación oportuno, logrando que el paciente se desempeñe en sus diferentes actividades cotidianas.

- El papel del fisioterapeuta especialista en neurorehabilitación es importante para el manejo integral de este tipo de pacientes durante todo el proceso de rehabilitación, ya que puede trabajar con el paciente no solo en su rehabilitación física, sino también integrar todos sus aspectos para mejorar en su parte senso-perceptiva, cognoscitiva y propioceptiva.

- Trabajar el input sensorial tras el concepto de sensorio-percepción nos permite evitar la neurodegeneración del sistema, formando así una programación central sensorio motora que permite en el paciente una recuperación integral y un estadio menor de los tiempos de hospitalización.

- Actualmente los estudios llevados a cabo por la Organización Mundial de la Salud (OMS) y el Banco Mundial señalan que hace falta contar con más profesionales y recursos destinados a la atención de personas con discapacidad (con especial énfasis en los niños).

- El proceso de neuromodulación del cerebro que se encuentra en maduración, permite activar los diferentes canales sensoriales a través de la estimulación de los sentidos (auditivo, visual, táctil, olfatorio), lo cual genera en el cerebro una respuesta favorable en el proceso de rehabilitación

- La ponente finaliza con un mensaje para sus asistentes "Para liberar el potencial propio, el secreto no está en la fuerza ni en la inteligencia, sino en el esfuerzo continuo".

\section{REFLEXIÓN FINAL}

\section{Cyndi Meneses $^{12}$}

Como coordinadora de la Especialización en Fisioterapia en Neurorehabilitación agradezco a todos los asistentes al seminario de Actualización en Neurorehabilitación en Pediatría por la acogida del evento, a los diferentes ponentes que quisieron compartir todos sus conocimientos y experticias en la neuropediatría, a los panelistas que a través de sus análisis generaron espacios enriquecedores, de cara a la actualización hacia un mundo que nos llama a ser mas preparadas, más competitivos, más expertos y sobre todo, a ser mejores seres humanos para quienes servimos y entregamos nuestra pasión en cada intervención.

Durante el evento se habló de cómo la integración sensorial toma fuerza para ser un complemento perfecto en el paciente pediátrico con TCE, cómo la neuropsicología hace parte fundamental de un proceso neurocognitivo en desarrollo, también, hemos visto cómo, partiendo de un adecuado análisis de la marcha podremos lograr mejores resultados en nuestros pacientes, sin dejar atrás la importancia que toma la actividad física y el deporte como una herramienta para

\footnotetext{
${ }_{12}$ Fisioterapeuta, Especialista y Magister en Neurorehabilitación de la Universidad Autónoma de Manizales, con 8 años de experiencia como Neurorehabilitadora y 2 años como Docente de pre y posgrado.
} 
trabajar las habilidades cognitivas en la infancia.

No podemos olvidar el proceso de evaluación que debe trabajar con gran esmero el neurorehabilitador, también, abordajes como la hidroterapia y alternativas de tratamiento que parten desde el enfoque neurorehabilitador, así como planteamientos de una nueva era en constante evolución donde modelos trabajados en animales puede darnos un control de las crisis epilépticas que nos dan la esperanza que en un futuro no muy lejano se pueda trabajar con mayor precisión en humanos. y por último la salud mental que es un tema que nos aborda en la actualidad desde la infancia del cual como neurorehabilitadores estamos llamados a ser actores principales para proteger la salud mental de nuestros niños.

Definitivamente queridos colegas, la invitación esta dada a continuar a lo que llamo el "mágico mundo de la neurorehabilitación", ese sendero hermosamente duro pero de grandes satisfacciones, que nos lleva a los contextos más profundos e inmersos de estrategias y abordajes terapéuticos donde la importancia del neurorehabilitador se convierte en un eje fundamental y prioritario hacia un camino de inclusión. 


\section{Referencias}

Ardila, A. \& Rosselli, M. (2007. Neuropsicología Clínica. Bogotá, D.C., Colombia: Manual moderno.

Das, P., \& Horton, R. (2016). Physical activity-time to take it seriously and regularly. Lancet, 388(10051), 1254-5.

Glenn, A. L., \& Raine, A. (2014). Neurocriminology: implications for the punishment, prediction and prevention of criminal behaviour. Nature Reviews Neuroscience, 15(1), 54.

Javela González, J. J. (2012). Modulación serotonérgica de las crisis epilépticas temporales en un modelo de kindling químico (Doctoral dissertation, Universidad Complutense de Madrid, Servicio de Publicaciones).

Kitchenham, B. (2004). Procedures for performing systematic reviews. Keele, UK: Keele University,

Martin, J. (1981). The Halliwick Method. Physiotherapy, 67(10), 288-291.

Muralidharan, K., \& Prakash, N. (2017). Cycling to school: Increasing secondary school enrollment for girls in India. American Economic Journal: Applied Economics, 9(3), 321-50.

Perry, J., \& Burnfield, J. M. (2010). Gait Analysis Normal and Pathological function, 2nd. Thorofare, NJ: Slack Incorporated.

Pozaa, J., Gómez, C., Gutiérrez, B., Mendoza, N.Y. \& Horneroa, R.(2012). Effects of a multi-sensory environment on brain-injured patients: Assessment of spectral patterns. Revista Medical Engineering \& Physics, 35 (2). 365-375.

Shaw, J. A., \& DeForge, R. T. (2012). Physiotherapy as bricolage: Theorizing expert practice. Physiotherapy Theory and Practice, 28(6), 420-427.

Soto, C., Salinas,P.,Y, \& Hidalgo,P. (2013).Aspectos fundamentales en la Rehabilitación Post TEC en elpaciente adulto y pediátrico. Revista médica clínica condes, 25 (2), 303-313.

Yague Sebastian, M. del pilar (2005), Estimulación multisensorial en el trabajo del fisioterapeuta pediatrico. Revista de fisioterapia, $27(4), 228-38$ 